\title{
Melanoma of the Sinonasal Tract: Value of a Septate Pattern on Precontrast T1-Weighted MR Imaging
}

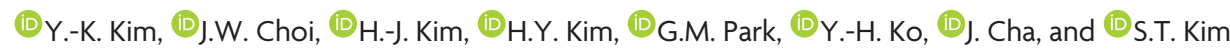

\begin{abstract}
BACKGROUND AND PURPOSE: Various tumors of the sinonasal tract can exhibit high signal intensity on TIWI. The purpose of this study was to determine the value of a septate pattern on precontrast TIWI for diagnosing sinonasal melanoma.
\end{abstract}

MATERIALS AND METHODS: Retrospectively, 3 observers independently reviewed MR images of 31 histologically proved sinonasal melanomas with special attention to the presence or absence of a septate pattern on precontrast T1WI, defined as alternating hyperintense and hypointense striations on precontrast TIWI. For comparison, we evaluated the prevalence of a septate pattern on precontrast TTWI in 106 nonmelanomatous sinonasal malignant tumors with 16 different histologic types. We also tried to identify the histopathologic features responsible for the septate pattern on precontrast TTWI.

RESULTS: Twenty-seven (87.1\%) of 31 sinonasal melanomas showed hyperintense foci on TIWI, among which a septate pattern on precontrast TIWI was seen in $23(74.2 \%)$, while 22 (20.8\%) of 106 nonmelanomatous malignant tumors demonstrated hyperintense foci on TTWI, among which only $3(2.8 \%)$ showed a septate pattern on precontrast TTWI. The sensitivity, specificity, positive predictive value, negative predictive value, and accuracy of a septate pattern on precontrast TIWI for the diagnosis of sinonasal melanoma were $74 \%$, $97 \%$, $88 \%, 93 \%$, and $92 \%$, respectively. Although limited due to the retrospective nature, 4 of 23 histologically reviewed sinonasal melanomas revealed an uneven distribution of melanin with alternating melanin and fibrous bands within the tumors.

CONCLUSIONS: A septate pattern on precontrast TIWI might be an adjunctive imaging finding for the diagnosis of sinonasal melanoma. This might be attributed histologically to an uneven distribution of melanin and hemorrhage within the tumors.

ABBREVIATIONS: SNM = sinonasal melanoma; TI-SP = septate pattern on precontrast Tl-weighted MR imaging

$\mathrm{M}_{\mathrm{a}}^{\mathrm{s}}$ elanoma of the sinonasal tract roughly accounts for $<1 \%$ of all melanomas and up to $4 \%$ of all sinonasal malignancies. ${ }^{1-5}$ According to the Surveillance, Epidemiology, and End Results data base, the approximate incidence of mucosal melanoma of the head and neck was $0.6-1.2$ cases per million persons per year from 1987 to 2009 in the United States. ${ }^{6}$ Of these, $72.6 \%$ of patients had disease in the sinonasal location. ${ }^{6}$ The incidence of

Received July 24, 2017; accepted after revision November 20

From the Departments of Radiology (Y.-K.K., H.-J.K., H.Y.K., G.M.P., J.C., S.T.K.) and Pathology (Y.-H.K.), Samsung Medical Center, Sungkyunkwan University School of Medicine, Seoul, Korea; Department of Radiology (J.W.C.), Ajou University School of Medicine, Suwon, Korea; Department of Radiology (G.M.P.), Ulsan University Hospital, University of Ulsan College of Medicine, Ulsan, Korea; and Department of Radiology and Research Institute of Radiological Science (I.C.), College of Medicine, Yonsei University College of Medicine, Seoul, Korea.

Yi-Kyung Kim and Jin Wook Choi contributed equally to this work.

Please address correspondence to Hyung-Jin Kim, MD, Department of Radiology, Samsung Medical Center, Sungkyunkwan University School of Medicine, 81 Irwonro, Gangnam-gu, Seoul 135-710, Korea; e-mail: hyungkim@skku.edu

http://dx.doi.org/10.3174/ajnr.A5539 mucosal melanoma appears to be increasing, especially in the sinonasal tract. ${ }^{6,7}$

Previous studies described high signal intensity on T1WI and low signal intensity on T2WI as the characteristic MR imaging features of melanoma. The signal characteristics on MR images are attributed to the paramagnetic properties of melanin pigment. ${ }^{8-13}$ However, several substances besides melanin, such as hemorrhage, high proteinaceous secretions, and fungus can also generate high signal intensity on T1WI. ${ }^{12,14}$ In some respects, sinonasal melanoma (SNM) is distinct from other malignant sinonasal tumors. First, the prognosis of SNM is reportedly much worse than other malignant sinonasal tumors, ${ }^{15-17}$ with its overall 5 -year survival rates being estimated at $<30 \%$ in most series, ${ }^{3}$ while those of other sinonasal malignancies range from $22 \%$ to $67 \%$, with an average of $45.5 \% .{ }^{16}$ Since 2010, the American Joint Committee on Cancer has created a separate system for staging mucosal melanoma of the head and neck, in which a primary tumor limited to the mucosa is considered T3, which reflects the aggressive biologic behavior of the tumor. ${ }^{18}$ Second, SNM 
Table 1: Visualization of a T1-SP on MR imaging in sinonasal melanomas and nonmelanomatous malignant sinonasal tumors based on consensus reading ${ }^{\text {a }}$

\begin{tabular}{|c|c|c|c|c|}
\hline & \multirow[b]{3}{*}{ Total } & \multicolumn{3}{|c|}{ Hyperintense Foci on TIWI } \\
\hline & & \multicolumn{2}{|c|}{ Present } & \multirow[b]{2}{*}{ Absent } \\
\hline & & T1-SP (+) & $\mathrm{TI}-\mathrm{SP}(-)$ & \\
\hline Sinonasal melanomab & 31 & 23 & 4 & 4 \\
\hline Nonmelanomatous malignant tumor ${ }^{b}$ & 106 & 3 & 19 & 84 \\
\hline Squamous cell carcinoma & 45 & 2 & 6 & 37 \\
\hline Lymphoma & 22 & 1 & 2 & 19 \\
\hline Adenoid cystic carcinoma & 10 & 0 & 4 & 6 \\
\hline Rhabdomyosarcoma & 5 & 0 & 0 & 5 \\
\hline Neuroendocrine carcinoma & 4 & 0 & 2 & 2 \\
\hline Adenocarcinoma & 4 & 0 & 2 & 2 \\
\hline Malignant fibrous histiocytoma & 3 & 0 & 1 & 2 \\
\hline Poorly differentiated carcinoma & 3 & 0 & 0 & 3 \\
\hline Spindle cell sarcoma & 3 & 0 & 0 & 3 \\
\hline Esthesioneuroblastoma & 1 & 0 & 0 & 1 \\
\hline Small round cell sarcoma & 1 & 0 & 1 & 0 \\
\hline Inflammatory myofibroblastic sarcoma & 1 & 0 & 0 & 1 \\
\hline Malignant peripheral nerve sheath tumor & 1 & 0 & 0 & 1 \\
\hline Ewing sarcoma & 1 & 0 & 1 & 0 \\
\hline Chondrosarcoma & 1 & 0 & 0 & 1 \\
\hline Myoepithelial carcinoma & 1 & 0 & 0 & 1 \\
\hline
\end{tabular}

Note:-_ indicates presence; - , absence.

a Data are presented as number of tumors.

${ }^{\mathrm{b}} P$ value $<.001$ by the Fisher exact test.

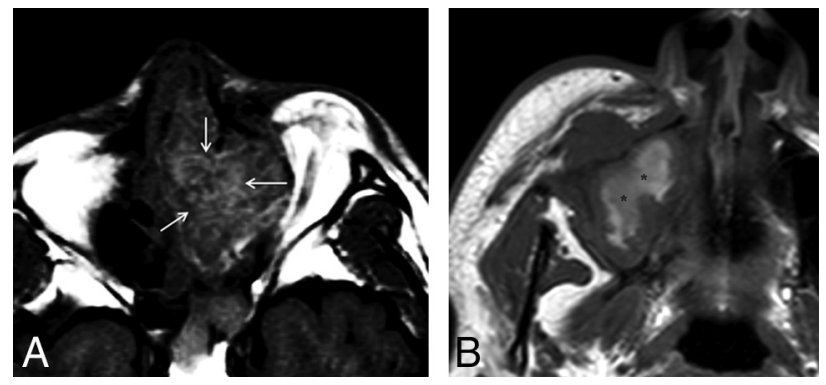

FIG 1. Two different types of SNM containing high signal intensity on TIWI with the presence $(A)$ or absence $(B)$ of a T1-SP. Although both tumors have intrinsic high signal intensity, only $A$ demonstrates a regular pattern of the alternating hyperintense and hypointense striations, so-called T7-SP (arrows). In contrast, the high signal intensity in $B$ appears amorphous without the alternating hyperintense and hypointense striations in a regular pattern (asterisks).

frequently presents as a mass with multicentric distribution. ${ }^{19}$ Third, promising results are constantly being reported on the efficacy of targeted therapy for treating advanced SNMs, including biochemotherapy using cytotoxic chemotherapy and biologic immunomodulatory agents such as interferon $\alpha$ and interleukin 2. ${ }^{3,20,21}$ The purpose of this study was to evaluate the diagnostic value of the septate pattern on precontrast T1-weighted MR imaging (T1-SP) for distinguishing SNMs from nonmelanomatous malignant sinonasal tumors.

\section{MATERIALS AND METHODS \\ Patients}

This study was approved by the institutional review board at Samsung Medical Center, and informed consent was waived in accordance with the requirements of a retrospective study.

Between January 1998 and January 2015, a search of the electronic medical records of our hospital revealed 56 patients (male/ female ratio $=34: 22$; mean age, 62 years; range, $21-88$ years) with histologically proved SNM, among whom MR imaging was performed in 35. Four patients were excluded from the study because no mass could be localized on MR images, resulting in 31 patients who were subjects of this study. There were 18 men and 13 women ranging in age from 37 to 88 years (mean age, 64 years).

To validate the role of T1-SP for diagnosing SNMs, we selected MR images of 106 adult patients with 16 different types of nonmelanomatous malignant sinonasal tumors through a search of the electronic data base of our institution between January 2010 and December 2014 using the keywords "malignant tumor of the nose and paranasal sinuses." All tumors were diagnosed histologically by biopsy and/or an operation. These 106 patients consisted of 63 males and 43 females with ages ranging from 14 to 87 years (mean age, 56 years). Table 1 demonstrates the pathologic subtypes, of which squamous cell carcinoma was most common $(n=45)$, followed by lymphoma $(n=22)$, adenoid cystic carcinoma $(n=10)$, and other sinonasal malignancies $(n=29)$.

\section{MR Imaging}

MR imaging examinations were performed on a 1.5T (Signa Advantage or Horizon; GE Healthcare, Milwaukee, Wisconsin) or 3T (Intera Achieva; Philips Healthcare, Best, the Netherlands) scanner. In all patients, precontrast spin-echo TIWI (TR/TE/ NEX, 400-560 ms/10-14 ms/2) and fast spin-echo T2WI (TR/ TE/NEX, 2500-4500 ms/80-110 ms/1) with or without fat saturation were obtained, followed by contrast-enhanced spin-echo T1WI with fat saturation after the intravenous injection of 0.1 $\mathrm{mmol} / \mathrm{kg}$ of gadolinium-based contrast material. Images were obtained in at least 2 planes with 3- to 4-mm section thickness and 0 to $1-\mathrm{mm}$ intersection gap.

\section{Image Analysis}

All MR images were evaluated by 3 radiologists with 4, 2, and 2 years of experience in neuroradiology, respectively, in an anonymized and randomized manner. All reviewers were blinded to the final histologic diagnosis. We evaluated the general MR imaging features of SNMs, such as the signal intensity and enhancement pattern. We determined the presence or absence of a T1-SP on MR imaging in 31 SNMs and 106 nonmelanomatous malignant sinonasal tumors. A T1-SP was determined to be present if a regular pattern of the alternating hyperintense and hypointense striations was distributed partially or diffusely within the solid components of the tumor on unenhanced T1WI (Fig 1A). Hyperintense foci without a regular pattern of alternating striations were not considered a T1-SP (Fig 1B). Before image review, the observers were instructed and tested on the imaging appearance 


\begin{tabular}{|c|c|c|c|c|c|c|c|c|}
\hline & \multicolumn{8}{|c|}{ Presence or Absence of T1-SP } \\
\hline & \multicolumn{2}{|c|}{ Observer 1} & \multicolumn{2}{|c|}{ Observer 2} & \multicolumn{2}{|c|}{ Observer 3} & \multicolumn{2}{|c|}{ Overall $^{b}$} \\
\hline & + & - & + & - & + & - & + & - \\
\hline Sinonasal melanoma & 22 & 9 & 26 & 5 & 20 & 11 & 23 & 8 \\
\hline Nonmelanomatous malignant sinonasal tumors & 7 & 99 & 1 & 105 & 3 & 103 & 3 & 103 \\
\hline
\end{tabular}

Note:- + indicates presence; -, absence.

${ }^{a}$ Interobserver agreement between observers 1 and $2, \kappa=.69$; between observers 1 and $3, \kappa=0.72$; between observers 2 and $3, \kappa=0.71$; average, $\kappa=0.71$.

${ }^{b}$ Data were obtained by consensus interpretation of 3 observers.

of a T1-SP. MR imaging interpretation proceeded in 2 ways: First, each observer independently reviewed the images in a randomized fashion; and second, the 3 observers reached a consensus by a joint interpretation that followed individual interpretation 1 month later to minimize recall bias.

\section{Histopathologic Evaluation}

Histopathologic slides were available for review in 23 of 31 SNMs. A board-certified pathologist with 30 years of experience in head and neck pathology retrospectively re-examined the pathologic slides with special attention to the presence or absence of melanin, the presence or absence of intratumoral hemorrhage, and the predominant cell type. The tumors were grouped into melanotic and amelanotic tumors with the former being further categorized as those with abundant melanin when the melanin-containing cells exceeded $10 \%$ of tumor cells and those with a moderate amount of melanin when they composed $<10 \%$. Finally, we tried to identify the histopathologic features responsible for T1-SP.

\section{Statistical Analysis}

On the basis of the observations made by each observer, interobserver agreement among 3 observers was evaluated by calculating $\kappa$ statistics. A $\kappa$ value $\leq 0.20$ indicated positive but poor agreement; $0.21-0.40$, fair agreement; $0.41-0.60$, moderate agreement; $0.61-0.80$, good agreement; and $\geq 0.81$, excellent agreement. On the basis of the results made by the 3 observers' consensus interpretations, the statistical difference of a T1-SP between SNMs and other malignant sinonasal tumors was analyzed using the Fisher exact test. We also determined the overall sensitivity, specificity, positive predictive value, negative predictive value, and accuracy of a T1-SP as the diagnostic indicator of SNM. The statistical differences in the prevalence of a T1-SP were sought according to the presence or absence of melanin, the presence or absence of hemorrhage, and the different cell types using the $\chi^{2}$ test. During statistical analysis, differences of $P<.05$ were considered statistically significant.

\section{RESULTS}

Compared with the brain stem, 26 of 31 SNMs showed heterogeneous isointense and hyperintense signal intensity on T1WI, while 5 tumors demonstrated homogeneous hypointense $(n=1)$ or heterogeneous isointense and hyperintense $(n=4)$ signal intensity. Various signal intensities were demonstrated on T2WI, including heterogeneous isointense and hypointense signal intensity $(n=15)$, heterogeneous isointense and hyperintense signal intensity $(n=9)$, and heterogenous hypointense and hyperintense signal intensity $(n=7)$. Twenty-seven tumors were generally well-enhanced on contrast-enhanced T1WI, while the re-

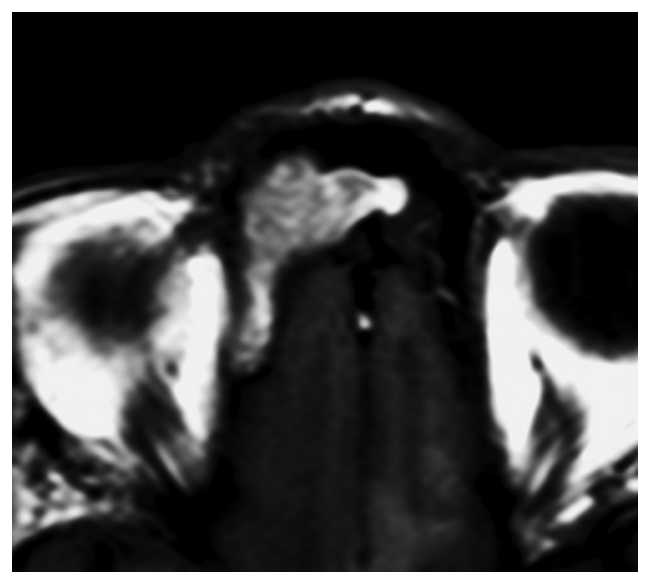

FIG 2. SNM in the right frontal sinus displaying a diffuse TI-SP. Precontrast TIWI demonstrates a mass with the alternating hyperintense and hypointense bands, the so-called TI-SP.

maining 4 tumors showed poor enhancement. Only 2 tumors demonstrated intratumoral necrosis.

\section{Comparison of the Prevalences of T1-SP between SNMs and Other Malignant Tumors}

On the basis of the results of individual and consensus interpretations, we summarized the prevalences of a T1-SP in SNMs and other nonmelanomatous sinonasal malignant tumors in Tables 1 and 2. Interobserver agreement among 3 observers for the presence of a T1-SP was good, with an average $\kappa$ value of 0.71 .

According to a consensus reading by the 3 observers, there was a significant statistical difference in the prevalence of a T1-SP between SNMs and other malignant tumors $(P<.001)$. Twentyseven $(87.1 \%)$ of 31 SNMs showed hyperintense foci on T1WI, among which a T1-SP was seen in $23(74.2 \%)$, either diffusely $(n=7$, Fig 2$)$ or partially $(n=16$, Fig 3$)$. In contrast, $22(20.8 \%)$ of 106 nonmelanomatous malignant sinonasal tumors demonstrated hyperintense foci on T1WI, among which only $3(2.8 \%)$ showed a T1-SP. These 3 tumors included 2 of 45 squamous cell carcinomas (Fig 4A) and 1 of 14 lymphomas (Fig 4B). Overall, the sensitivity, specificity, positive predictive value, negative predictive value, and accuracy of a T1-SP for the diagnosis of SNM were 74\% (95\% CI, 55\%-88\%), 97\% (95\% CI, 92\%-99\%), 88\% (95\% CI, 71\%-96\%), 93\% (95\% CI, 88\%-96\%), and 92\% (95\% CI, $83 \%-96 \%)$, respectively.

\section{Histopathologic Features of SNMs in Correlation with MR Imaging Features}

Of 23 SNMs for which retrospective histopathologic analysis was available, 18 tumors were melanotic and 5 were amelanotic. Overall, intratumoral hemorrhage was present in 14 and absent in 9. 

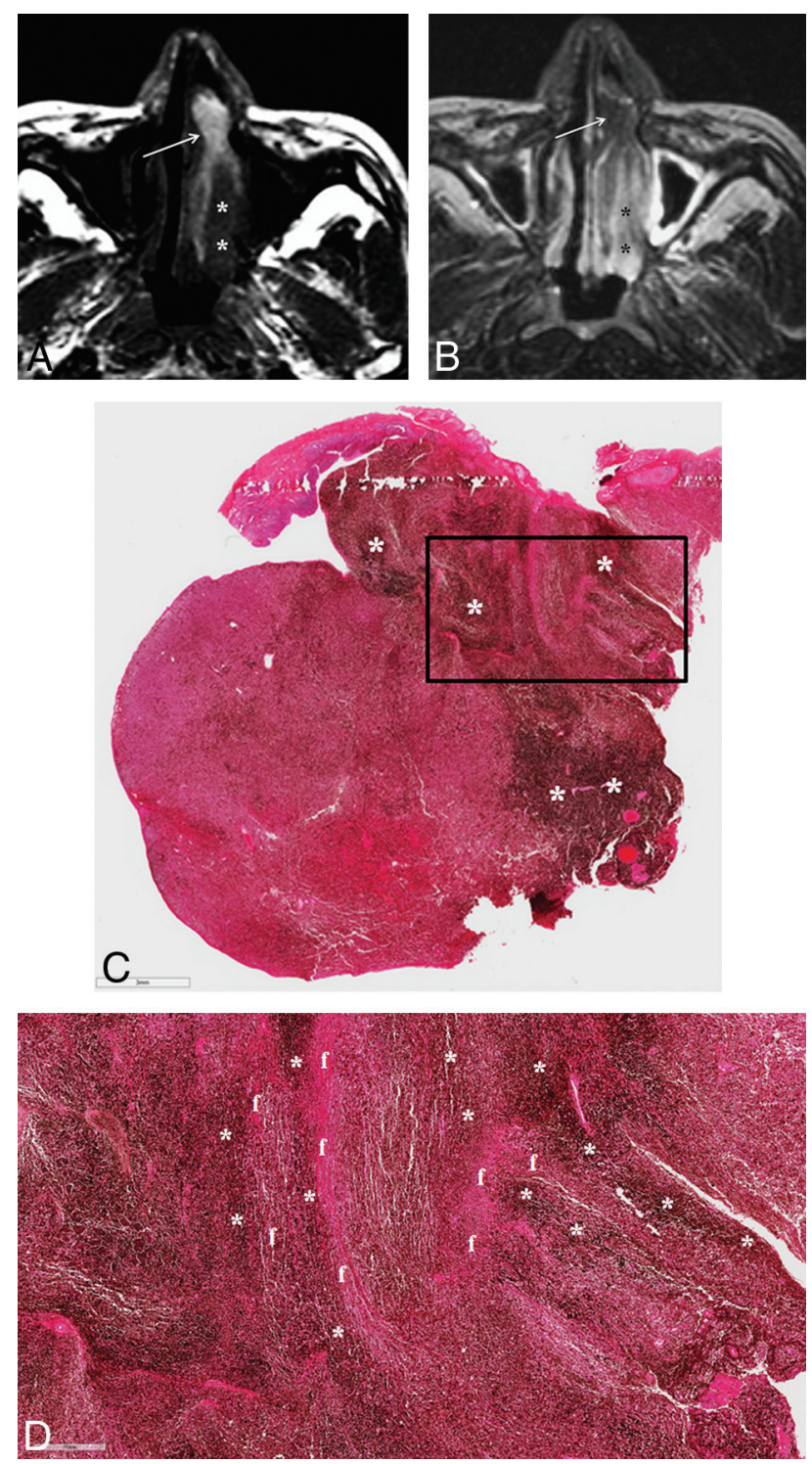

FIG 3. SNM displaying a partial TI-SP. A, Precontrast axial TIWI demonstrates an elongated mass with heterogeneous signal intensity in the left nasal cavity. While the anterior portion of the mass shows the alternating hyperintense and hypointense bands, the so-called TT-SP (arrow), the posterior portion is the soft-tissue component with the signal intensity isointense to the brain stem (asterisks). B, On fatsuppressed axial T2WI, the anterior portion of the mass appears isointense to the brain stem (arrow), while the posterior portion becomes hyperintense (asterisks). Insufficient facial and buccal fat suppression was caused by magnetic field inhomogeneity due to metallic dental hardware. C. Photomicrograph reveals an uneven distribution of melanin pigments (dark bands marked with asterisks). The bar on the left bottom indicates $3 \mathrm{~mm}$ (hematoxylin-eosin, original magnification $\times 7)$. D, Photomicrograph with higher magnification corresponding to the box in $C$ shows the area of the alternating melanin (dark bands marked with asterisks) and fibrous (f) bands. The bar on the left bottom indicates $700 \mu \mathrm{m}$ (hematoxylin-eosin, original magnification $\times 30)$.

Various histologic cell types were seen, including 15 epithelioid cell, 3 spindle cell, and 5 mixed cell types. Correlation of the histopathologic features with the MR imaging features in these 23 SNMs in terms of the presence or absence of a T1-SP is summarized in Table 3. A T1-SP was found in 14 of 18 melanotic melanomas and 4 of 5 amelanotic melanomas. Twelve of 14 tumors

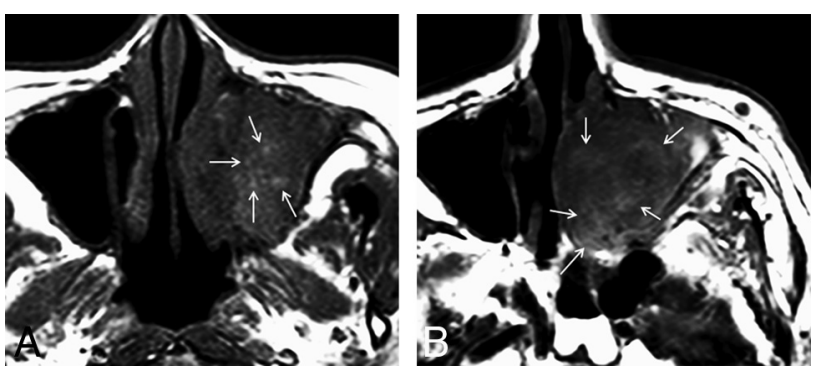

FIG 4. Examples of nonmelanomatous sinonasal tumors displaying a T1-SP (arrows). A, Squamous cell carcinoma. B, Lymphoma.

Table 3: Correlation of histopathologic and MR imaging features of 23 sinonasal melanomas ${ }^{\mathrm{a}}$

\begin{tabular}{lccc}
\hline & T1-SP (+) & T1-SP (-) & P Value $^{\mathbf{b}}$ \\
\hline Melanin & & & .915 \\
Melanotic & 14 & 4 & $.043^{\mathrm{C}}$ \\
Abundant & 9 & 0 & \\
Moderate & 5 & 3 & \\
Amelanotic & 4 & 1 & .280 \\
Hemorrhage & & & \\
Present & 12 & 2 & .399 \\
Absent & 6 & 3 & \\
Cell type & & & \\
Epithelioid & 12 & 3 & \\
Spindle & 3 & 0 & \\
Mixed & 3 & 2 & \\
\hline
\end{tabular}

Note:- + indicates presence; -, absence.

${ }^{a}$ Data are presented as numbers of tumors.

${ }^{b}$ Comparison of the prevalence of a TT-SP according to the presence of melanin, the presence of hemorrhage, and the different cell types using the $\chi^{2}$ test.

${ }^{\mathrm{C}}$ Comparison of the prevalence of a T-SP between tumors with abundant melanin and the group of tumors with no and moderate melanin by the $\chi^{2}$ test.

with intratumoral hemorrhage had a T1-SP, while 6 of 9 tumors without hemorrhage demonstrated it. There was no significant statistical difference in the prevalence of a T1-SP among tumors regarding the presence of melanin $(P=.915)$, the presence of hemorrhage $(P=.280)$, and the different cell types $(P=.399)$. Melanin pigments were abundant in 9 of 18 melanotic tumors and moderate in the remaining 9 tumors. If melanotic tumors with moderate melanin and amelanotic tumors were grouped together, there was a significant statistical difference in the prevalence of a T1-SP between the group of tumors with no and moderate melanin and melanotic tumors with abundant melanin $(P=$ .043 using the $\chi^{2}$ test), suggestive of a close relationship between the amount of melanin and the presence of T1-SP. Hemorrhage was present in 10 of 18 melanotic tumors and 4 of 5 amelanotic tumors. T1-SP was demonstrated in 1 amelanotic melanoma that had no hemorrhage on histologic examination.

Although the retrospective nature of this study kept us from performing a side-by-side MR imaging-pathologic correlation, 4 cases revealed an uneven distribution of melanin with alternating melanin and fibrous bands, which might be considered one of the pathologic features responsible for a T1-SP on MR imaging (Fig $3 C,-D)$.

\section{DISCUSSION}

The results of the present study are promising in that a T1-SP might be a useful imaging marker for the diagnosis of SNM with high specificity (97\%) and a moderate sensitivity (74\%). While 23 
(74.2\%) of 31 SNMs showed a T1-SP, only 3 (2.8\%) of 106 nonmelanomatous malignant tumors demonstrated it. It also proved to be reproducible, shown by good interobserver agreement.

In this study, 14 of 18 melanotic SNMs and 4 of 5 amelanotic SNMs demonstrated a T1-SP. Although we failed to reveal a significant statistical difference in the prevalence of a T1-SP between melanotic and amelanotic tumors, our results showing a significant higher prevalence of a T1-SP in SNMs compared with other nonmelanomatous tumors may still be good evidence that melanin pigment is the important source for T1-SP. In their study with 7 melanotic SMMs and 5 amelanotic SMMs, Yousem et $\mathrm{al}^{11}$ reported that all melanotic tumors were hyperintense to gray matter on T1WI regardless of the presence of hemorrhage, while the signal intensities of all amelanotic tumors were intermediate on T1WI. They suggested that the presence of melanin should be considered the main cause of hyperintensity. ${ }^{11}$

We also failed to find any statistical significance of the prevalence of a T1-SP between hemorrhagic and nonhemorrhagic tumors and among tumors of the different cell types as well. In their study with 6 melanotic SNMs and 5 amelanotic SNMs, Kim et $\mathrm{al}^{9}$ suggested that the signal intensity of SNMs on MR imaging was affected mainly by melanin pigments but also, in part, by the hemorrhagic products. We speculate that intratumoral hemorrhage in addition to melanin should play a role in creating a T1-SP in a certain proportion of SNMs, as seen in 4 amelanotic SNMs and 3 nonmelanomatous sinonasal tumors demonstrating a T1-SP in this study. Most interesting, we found an intimate relationship between the amount of melanin and the presence of a T1-SP. Although no statistical difference in the prevalence of a T1-SP was found between melanotic and amelanotic tumors, the difference was significant between tumors with abundant melanin and the group of tumors with no and moderate melanin. This finding is in accordance with the results of the study by Kim et al, ${ }^{9}$ who reported that the signal intensity pattern could vary on MR imaging depending on the amount and distribution of melanin within individual SNMs.

Previous studies also found intratumoral vessels and fibrous septa within malignant melanomas on histologic examination. ${ }^{9,22}$ We suggest that a T1-SP may be attributed to an uneven distribution of melanin and hemorrhage within the tumors. In this study, 1 amelanotic tumor without evidence of hemorrhage demonstrated a T1-SP. Although unclear, it is possible that insufficient histopathologic examination might have overlooked the presence of melanin and/or hemorrhage in this case. Although an uneven distribution of melanin with the alternating melanin and fibrous bands within the tumors seen in 4 cases in this study might be considered one of the pathologic features responsible for a T1-SP, the retrospective nature of this study interfered with an exact 1:1 MR imaging-pathologic correlation; thus, the elucidation of the exact histopathologic basis for a T1-SP was difficult. We recommend a prospective study focusing on the MR imaging-pathologic correlation in a large cohort of patients with SNM in the near future.

A T1-SP seen in an SNM in the present study looks like the convoluted cerebriform pattern seen in inverted papillomas. ${ }^{23}$ The only difference is that the former is best seen on T1WI, while the latter is seen on T2WI and contrast-enhanced T1WI. A "con- voluted cerebriform pattern" is the term first used in the pathology literature by Barnes et $\mathrm{al}^{24}$ to describe a distinctive gross mucosal morphology of inverted papilloma, created by the juxtaposed epithelial and stromal layers. This peculiar mucosal morphology results in a characteristic pattern on MR imaging (ie, the alternating hypointense and hyperintense bands on T2-weighted and contrast-enhanced T1-weighted images), making the imaging diagnosis of inverted papilloma possible. ${ }^{23,25}$ Histologically, the convoluted cerebriform pattern seen in inverted papilloma is known to result from the alternation of highly cellular metaplastic epithelium and less cellular edematous stroma. ${ }^{25}$

This study has several limitations. First, the case series of SNMs is small, and the number of SNMs that underwent a histopathologic review is even smaller. Second, we used varying imaging parameters and different magnets in this study. These might alter the results of the prevalence of T1-SP because a higher magnetic field strength causes a greater sensitivity to T1 shortening. Third, the histopathologic features responsible for T1-SP were not exactly determined because the retrospective nature of this study prevented us from performing histopathologic re-examination in all cases of SNM, making an exact 1:1 MR imaging-pathologic correlation difficult. Fourth, the proportion of SNMs included in this study is roughly $23 \%$ (31 of 137), which is much higher than up to $4 \%$ reported among all sinonasal malignant tumors. The skewed composition of the patients due to the unusually high proportion with the disease of interest can have an unreliable impact on the calculations of the diagnostic index, such as specificity, positive and negative predictive values, and accuracy and thus may not reflect the true values encountered in clinical practice.

\section{CONCLUSIONS}

Despite several limitations of this study, a T1-SP might be an adjunctive MR imaging finding for distinguishing SNM from various nonmelanomatous malignant sinonasal tumors with an overall accuracy of $92 \%$. Although the exact histopathologic features responsible for a T1-SP are yet to be determined, we suggest that it may be attributed to an uneven distribution of melanin and hemorrhage within the tumors. A prospective study focusing on the MR imaging-pathologic correlation is highly recommended.

\section{REFERENCES}

1. Chang AE, Karnell LH, Menck HR. The National Cancer Data Base report on cutaneous and noncutaneous melanoma: a summary of 84,836 cases from the past decade: the American College of Surgeons Commission on Cancer and the American Cancer Society. Cancer 1998;83:1664-78 Medline

2. Bachar G, Loh KS, O'Sullivan B, et al. Mucosal melanomas of the head and neck: experience of the Princess Margaret Hospital. Head Neck 2008;30:1325-31 CrossRef Medline

3. López F, Rodrigo JP, Cardesa A, et al. Update on primary head and neck mucosal melanoma. Head Neck 2016;38:147-55 CrossRef Medline

4. Moreno MA, Roberts DB, Kupferman ME, et al. Mucosal melanoma of the nose and paranasal sinuses, a contemporary experience from the M. D. Anderson Cancer Center. Cancer 2010;116:2215-23 CrossRef Medline

5. Lombardi D, Bottazzoli M, Turri-Zanoni M, et al. Sinonasal mucosal melanoma: a 12-year experience of $\mathbf{5 8}$ cases. Head Neck 2016; 38(suppl 1):E1737-45 CrossRef Medline 
6. Marcus DM, Marcus RP, Prabhu RS, et al. Rising incidence of mucosal melanoma of the head and neck in the United States. J Skin Cancer 2012;2012:231693 Medline

7. Youssef D, Vasani S, Marquess J, et al. Rising incidence of head and neck mucosal melanoma in Australia. J Laryngol Otol 2017;131: S25-28 CrossRef Medline

8. Batra K, Chhabra A, Rampure J, et al. CT and MRI appearances of primary sphenoid melanoma: a rare case. AJNR Am J Neuroradiol 2005;26:2642-44 Medline

9. Kim SS, Han MH, Kim JE, et al. Malignant melanoma of the sinonasal cavity: explanation of magnetic resonance signal intensities with histopathologic characteristics. Am J Otolaryngol 2000;21: 366-78 CrossRef Medline

10. Xu QG, Fu LP, Wang ZC, et al. Characteristic findings of malignant melanoma in the sinonasal cavity on magnetic resonance imaging. Chin Med J (Engl) 2012;125:3687-91 Medline

11. Yousem DM, Li C, Montone KT, et al. Primary malignant melanoma of the sinonasal cavity: MR imaging evaluation. Radiographics 1996; 16:1101-10 CrossRef Medline

12. Escott EJ. A variety of appearances of malignant melanoma in the head: a review. Radiographics 2001;21:625-39 CrossRef Medline

13. Enochs WS, Petherick P, Bogdanova A, et al. Paramagnetic metal scavenging by melanin: MR imaging. Radiology 1997;204:417-23 CrossRef Medline

14. Woodruff WW Jr, Djang WT, McLendon RE, et al. Intracerebral malignant melanoma: high-field-strength MR imaging. Radiology 1987;165:209-13 CrossRef Medline

15. Turner JH, Reh DD. Incidence and survival in patients with sinonasal cancer: a historical analysis of population-based data. Head Neck 2012;34:877-85 CrossRef Medline

16. Khademi B, Moradi A, Hoseini S, et al. Malignant neoplasms of the sinonasal tract: report of 71 patients and literature review and analysis. Oral Maxillofac Surg 2009;13:191-99 CrossRef Medline

17. Bhattacharyya N. Factors affecting survival in maxillary sinus cancer. J Oral Maxillofac Surg 2003;61:1016-21 CrossRef Medline

18. Edge SB, Compton CC. The American Joint Committee on Cancer: the 7th edition of the AJCC cancer staging manual and the future of TNM. Ann Surg Oncol 2010;17:1471-74 CrossRef Medline

19. Stanimirov Rossi O, Vital D, Soyka MB, et al. Multilocular sinonasal malignant melanoma: a poor prognostic subgroup? Eur Arch Otorhinolaryngol 2015;272:123-29 CrossRef Medline

20. Bartell HL, Bedikian AY, Papadopoulos NE, et al. Biochemotherapy in patients with advanced head and neck mucosal melanoma. Head Neck 2008;30:1592-98 CrossRef Medline

21. Bedikian AY, Johnson MM, Warneke CL, et al. Prognostic factors that determine the long-term survival of patients with unresectable metastatic melanoma. Cancer Invest 2008;26:624-33 CrossRef Medline

22. Nazarian LN, Alexander AA, Kurtz AB, et al. Superficial melanoma metastases: appearances on gray-scale and color Doppler sonography. AJR Am J Roentgenol 1998;170:459-63 CrossRef Medline

23. Jeon TY, Kim HJ, Chung SK, et al. Sinonasal inverted papilloma: value of convoluted cerebriform pattern on MR imaging. AJNR Am J Neuroradiol 2008;29:1556-60 CrossRef Medline

24. Barnes L, Verbin RS, Gnepp DR. Diseases of the nose, paranasal sinuses, and nasopharynx. In: Barnes L, ed. Surgical Pathology of the Head and Neck. Vol 1. New York: Marcel Dekker; 1985:403-51

25. Ojiri H, Ujita M, Tada S, et al. Potentially distinctive features of sinonasal inverted papilloma on MR imaging. AJR Am J Roentgenol 2000;175:465-68 CrossRef Medline 